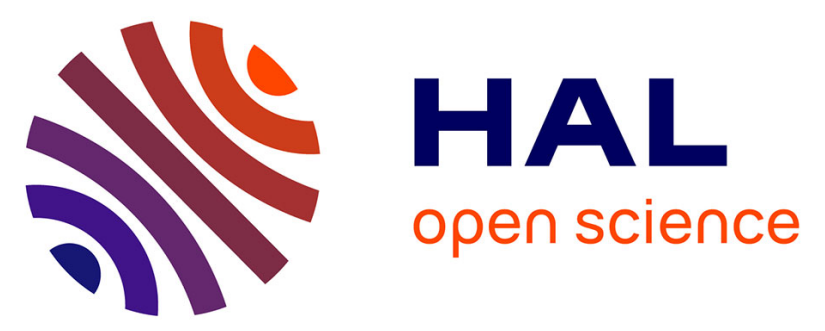

\title{
Further remarks on the effect of multiple spectral values on the dynamics of time-delay systems. Application to the control of a mechanical system
}

Islam Boussaada, Sami Tliba, Silviu-Iulian Niculescu, Hakki Ulaş Unal, Tomas Vyhlidal

\section{To cite this version:}

Islam Boussaada, Sami Tliba, Silviu-Iulian Niculescu, Hakki Ulaş Unal, Tomas Vyhlidal. Further remarks on the effect of multiple spectral values on the dynamics of time-delay systems. Application to the control of a mechanical system. Linear Algebra and its Applications, 2018, 542, pp.589-604. 10.1016/j.laa.2017.11.022 . hal-01657659

\section{HAL Id: hal-01657659}

https://hal-centralesupelec.archives-ouvertes.fr/hal-01657659

Submitted on 10 Jan 2018

HAL is a multi-disciplinary open access archive for the deposit and dissemination of scientific research documents, whether they are published or not. The documents may come from teaching and research institutions in France or abroad, or from public or private research centers.
L'archive ouverte pluridisciplinaire HAL, est destinée au dépôt et à la diffusion de documents scientifiques de niveau recherche, publiés ou non, émanant des établissements d'enseignement et de recherche français ou étrangers, des laboratoires publics ou privés. 


\title{
Further Remarks on the Effect of Multiple Spectral Values on the Dynamics of Time-delay Systems. Application to the control of a Mechanical system
}

\author{
Islam Boussaada $^{\mathrm{a}, \mathrm{b}}$, Sami Tliba ${ }^{\mathrm{a}}$, Silviu-Iulian Niculescu ${ }^{\mathrm{a}}$, Hakki Ulaş Ünal ${ }^{\mathrm{c}}$, Tomáš Vyhlídal ${ }^{\mathrm{d}}$ \\ ${ }^{a}$ Laboratoire des Signaux et Systèmes, Université Paris Saclay, CNRS-Supélec-Université Paris Sud \\ 3 rue Joliot-Curie, 91192 Gif-sur-Yvette cedex (France) \\ ${ }^{b}$ Institut Polytechnique des Sciences Avancées \\ 7 rue Maurice Grandcoing, 94200 Ivry-sur-Seine (France) \\ ${ }^{c}$ Department of Electrical and Electronics Engineering, Anadolu University, Eskişehir, Turkey \\ ${ }^{d}$ Department of Instrumentation and Control Engineering, Czech Technical University in Prague, Czech Republic
}

\begin{abstract}
A question of ongoing interest for linear Time-delay systems is to determine conditions on the equation parameters that guarantee the exponential stability of solutions. In recent works a new interesting property of time-delay systems was emphasized. As a matter of fact, the multiple spectral values for time-delay systems was characterized by using a Birkhoff/Vandermondebased approach. Then, a multiplicity induced stability criteria exhibited for reduced order systems; scalar delay-equations and a special class of second order systems. This work, further explores such a criteria and shows their applicability to the control of a mechanical system.
\end{abstract}

Keywords: Time-Delay, Stability, frequency-domain approach, Control design, Vibration control

\section{Introduction}

${ }^{1}$ In this paper, the special class of nonlinear eigenvalue problems derived from the frequency domain analysis of Time-delay systems is considered. An ongoing interest for such an eigenvalue analysis is motivated by the wide range of applications where time-delays occurs. As a matter of fact, the asymptotic behavior of the solutions of this class of infinite dimensional dynamical systems is determined from the corresponding spectrum designating the set of the roots of the associated characteristic function often called quasipolynomial, that is a transcendental polynomial in the Laplace variable in which appear exponential terms induced by delays, see for instance [1]. The study of the zeros of such a class of entire functions [2] plays a crucial role especially in the analysis of the asymptotic stability of the zero solution associated with dynamical systems.

Email addresses: Islam. Boussaada@12s . centralesupelec.fr (Islam Boussaada),

Sami.Tliba@12s.centralesupelec.fr (Sami Tliba), Silviu.Niculescu@l2s.centralesupelec.fr

(Silviu-Iulian Niculescu), huunal@anadolu .edu.tr (Hakki Ulaş Ünal), tomas .vyhlidal@fs .cvut .cz (Tomás̆ Vyhlídal)

${ }^{1}$ The content of this paper was partially presented in The 20th Conference of the International Linear Algebra Society, July 11-15, 2016, KU Leuven, Belgium 
Indeed, the zero solution is asymptotically stable if all the spectral values are in the open lefthalf complex plane [3]. Furthermore, the crucial effect of multiplicities (algebraic/geometric) of a given spectral values on the stability of the steady-state of the corresponding dynamical system is well known, see for instance [3]. But, such multiple roots may produce complex behaviors, for instance, in the case of multiple Hopf points' dynamics one refers the reader to the work [4]. One of the main ingredients allowing to multiple spectral values is the sysmmetry. Symmetries in dynamical systems often induce equivariance conditions, which may be associated with multiple spectral values. It is observed in [5] that the existence of multi-dimensional irreducible representations of the symmetry group may force a spectral value to be multiple. Also, such multiple spectral values may occur in optimization problems, see for instance [6].

Recent works by the authors [7, 8, 9] characterized multiple Crossing Imaginary Roots (CIR) for time-delay systems using a Birkhoff/Vandermonde-based approach. In [8] it is shown that the admissible multiplicity of the zero spectral value is bounded by the generic Polya and Szegö bound denoted $P S_{B}$, which is nothing but the degree of the corresponding quasipolynomial, see for instance [10]. In [7] it is shown that a given CIR with non vanishing frequency never reaches $P S_{B}$ and a sharper bound for its admissible multiplicities is established. However, even the characterization of the multiplicity of a given complex (non real) spectral value may be carried out by the same approach, it involves hyperbolic/trigonometric functional confluent Vandemonde matrices. Moreover, in such a case the $P S_{B}$ can never be reached. Furthermore, an example of a scalar retarded equation with two delays is studied in [7] where it is shown that the multiplicity of real spectral values may reach the $P S_{B}$. The corresponding system has some further interesting properties: (i) it is asymptotically stable, (ii) its spectral abscissa (rightmost root) corresponds to this maximal allowable multiple root located on the imaginary axis. Such observations enhance the outlook of further exhibiting the existing links between the maximal allowable multiplicity of some negative spectral value reaching the quasipolynomial degree ${ }^{2}$ and the stability of the trivial solution of the corresponding dynamical system. Such a property was already observed in [11], where a tuning strategy is proposed for the design of a delayed Proportional-Integral controller by placing a triple real dominant root for the closed-loop system. However, the right-most position is only checked using a Mikhailov curve and QPmR toolbox, see for instance [12]. In the sequel, the above property which is called in [13] multiplicity induced stability will be further explored.

The present work is a natural continuation of $[7,8,13,14]$, it aims first to improve the understanding of the correspondence between the multiple spectral value variety and the stable variety associated with the steady state solution, and secondly, to demonstrate the applicability of such a property to a mechanical engineering problem.

The remaining paper is organized as follows. Section 2 introduces the prerequisite and states the problem. Also, some motivating reduced order examples are discussed. Next, the main results are enunciated and proved in Section 3, namely, the multiplicity induced stability is explored for the general second order delay equation. Section 4, is devoted to the application to the control of active vibrations. Concluding remarks end the paper.

\section{Problem Statement and Motivations}

In this section, second-order linear systems of retarded type are explored. Namely, a sufficient condition for the asymptotic stability of the following class of LTI time-delay systems is

${ }^{2}$ The quasipolynomial degree is nothing but the number of the involved polynomials plus their degree minus one 
presented. Consider the system

$$
\dot{x}=A_{0} x(t)+A_{1} x(t-\tau)
$$

with the state-vector $x=\left(x_{1}, x_{2}\right) \in \mathbb{R}^{2}$, under appropriate initial conditions belonging to the Banach space of continuous functions $C\left(\left[-\tau_{N}, 0\right], \mathbb{R}^{2}\right)$. Here $\tau$ is a positive constant delays and the matrices $A_{j} \in \mathcal{M}_{2}(\mathbb{R})$ for $j=0 \ldots 1$. It is well known that the asymptotic behavior of the solutions of (1) is determined from the spectrum $\boldsymbol{N}$ designating the set of the roots of the associated characteristic function (denoted in the sequel $\Delta(s, \tau)$ ). Namely, the characteristic function corresponding to system (1) is a quasipolynomial $\Delta: \mathbb{C} \times \mathbb{R}^{n}{ }_{+} \rightarrow \mathbb{C}$ of the form:

$$
\Delta(s, \tau)=\operatorname{det}\left(s I-A_{0}-A_{1} e^{-\tau s}\right) .
$$

To start with, let us recall recent results exploiting multiple spectral values to establish sufficient conditions for the asymptotic stability of the zero solution of system of type (1).

\subsection{Scalar equation with a delay}

Consider the simple scalar differential equation with one delay representing a biological model discussed by K. L. Cooke in [15]. It describes the dynamics of a vector disease dynamics where the infected host population $x(t)$ is governed by:

$$
\dot{x}(t)+a_{0} x(t)+a_{1} x\left(t-\tau_{1}\right)-a_{1} x\left(t-\tau_{1}\right) x(t)=0,
$$

here $a_{1}>0$ designates the contact rate between infected and uninfected populations and it is assumed that the infection of the host recovery proceeds exponentially at a rate $-a_{0}>0$, see also [16] for more insights on the modeling and stability results. Let us focus on the linearized system which is given by:

$$
\dot{x}(t)+a_{0} x(t)+a_{1} x(t-\tau)=0 .
$$

In [13], it is shown that for a given delay $\tau \in \mathbb{R}_{+}^{*}$, equation (4) admits a double spectral value at $z=z_{0}$ if and only if $z_{0}=-a_{0} \tau+1 / \tau$ and $a_{1}=\mathrm{e}^{-a_{0} \tau-1} / \tau$. If in addition, $a_{0}>-1 / \tau$ then the zero solution of system (4) is asymptotically stable and $z_{0}$ is nothing but the corresponding rightmost root.

\subsection{A particular second order delay equation}

In [13] the well known Sunflower model is considered. Namely, the helical movement of a growing plant is governed by the following delay equation:

$$
\ddot{x}+\frac{a}{\tau} \dot{x}+\frac{b}{\tau} \sin (x(t-\tau))=0
$$

Such a model is known to reproduce the dynamics of the upper part of the stem of the plant, which performs a rotating movement. Here the state $x(t)$ designates the angle of the plant with respect to the vertical line and the delay $\tau$ corresponds to a geotropic reaction time and $a$ and $b$ some positive parameters. The corresponding linearized system with $\alpha=a / \tau$ and $\beta=b / \tau$ is given by:

$$
\ddot{x}+\alpha \dot{x}+\beta x(t-\tau)=0
$$


In [13] it is shown that equation (6) admits a spectral value at $z$ with multiplicity 2 if and only if $(\alpha, z)=\left(\alpha_{+}, z_{+}\right)$or $(\alpha, z)=\left(\alpha_{-}, z_{-}\right)$where:

$$
\left\{\begin{array}{l}
\alpha_{-}=-\left(2+\sqrt{4+\tau^{2} \beta^{2}}\right) \mathrm{e}^{1 / 2 \tau\left(-\beta-\frac{2+\sqrt{4+\tau^{2} \beta^{2}}}{\tau}\right)} \tau^{-2}, \\
z_{-}=-\frac{\beta}{2}-\frac{2+\sqrt{4+\tau^{2} \beta^{2}}}{2 \tau} \\
\alpha_{+}=\left(-2+\sqrt{4+\tau^{2} \beta^{2}}\right) \mathrm{e}^{1 / 2 \tau\left(-\beta+\frac{-2+\sqrt{4+\tau^{2} \beta^{2}}}{\tau}\right)} \tau^{-2}, \\
z_{+}=-\frac{\beta}{2}+\frac{-2+\sqrt{4+\tau^{2} \beta^{2}}}{2 \tau}
\end{array}\right.
$$

It is also proven that if $(z, \alpha)=\left(z_{+}, \alpha_{+}\right)$(respectively $\left.\left(z_{-}, \alpha_{-}\right)\right)$and $\tau \beta>2 \sqrt{3}(\tau \beta<2 \sqrt{3})$ then $z_{+}$(respectively $z_{-}$) is a the rightmost root and the corresponding steady state solution is asymptotically stable. The next section enunciates the main contribution, which extends the above results.

\section{Rightmost Characteristic root assignment for the general second order equation with a single constant delay: Maximal multiplicity based approach}

On the one hand, second-order linear systems capture the dynamic behavior of many natural phenomena, and have found wide applications in a variety of fields, such as vibration and structural analysis. On the other hand, it is shown in [17] the stabilizing effect of the delay in the control, that is the closed-loop stability is guaranteed precisely due to the existence of the delay. Here, we are concerned by the problem of the analytical characterization of the rightmost root corresponding to the general second order time-delay systems where the control is a delayed state feedback. More precisely, we consider Time-delay system where the corresponding characteristic function is written as:

$$
\Delta(s, \tau)=s^{2}+c_{1} s+c_{0}+\beta e^{-\tau s} .
$$

Using the following change of variables

$$
s=\frac{c_{1} \lambda}{2}
$$

one obtains the corresponding normalized characteristic function:

$$
\tilde{\Delta}(\lambda, \tilde{\tau})=\lambda^{2}+2 \lambda+a_{0}+\alpha e^{-\lambda \tilde{\tau}}, \text { where } \alpha=\frac{4}{c_{1}^{2}} \beta, \tilde{\tau}=\frac{c_{1}}{2} \tau, a_{0}=4 \frac{c_{0}}{c_{1}{ }^{2}} .
$$

It is well known that if all $\alpha=0$ and the problem of spectral abscissa minimization is considered then $a_{0}=1$ and the rightmost root is $\lambda_{0}=-1$, see for instance [18]. By exploiting the delay effect, we are able to increase the solution's convergence rate by decreasing the corresponding rightmost root.

Let assume that $a_{0}>1$, then the following Theorem holds.

\section{Theorem 3.1.}


i) The multiplicity of any given root of the quasipolynomial function (10) is bounded by 3 .

ii) The quasipolynomial (10) admits a real spectral value at $\lambda=\lambda_{0}$ with algebraic multiplicity 3 if and only if

$$
\tilde{\tau}=\sqrt{\frac{1}{a_{0}-1}}, \lambda_{0}=-1-\frac{1}{\tilde{\tau}}, \alpha=-\frac{2 e^{-(1+\tilde{\tau})}}{\tilde{\tau}^{2}} .
$$

iii) If (11) is satisfied then $\lambda=\lambda_{0}$ is the rightmost root of (10).

Proof. Assertion i) follows directly from Pólya-Szegö Theorem presented in Appendix, see also [8, 9]. Indeed, setting $\alpha=\beta=0$ gives a bound of the number of real roots for (10), which is nothing but the degree of the quasipolynomial. The maximal number of real roots is itself a bound for the multiplicity of any real root. The vanishing of the corresponding quasipolynomial $\tilde{\Delta}(\lambda, \tilde{\tau})=0$ allows to isolate the exponential term

$$
e^{-\lambda \tau}=\frac{-a_{0}-2 \lambda-\lambda^{2}}{\alpha} .
$$

Since we are investigating the algebraic multiplicity three of roots of (10), one substites (12) in the first two derivatives, which allows to the system of algebraic equations:

$$
\left\{\begin{array}{l}
2+\tau_{1}^{2}\left(-a_{0}-2 \lambda-\lambda^{2}\right)=0 \\
2+2 \lambda-\tau_{1}\left(-a_{0}-2 \lambda-\lambda^{2}\right)=0
\end{array}\right.
$$

Solving (13) in $\lambda$ and $\tilde{\tau}$, gives the unique solution $\tilde{\tau}=\sqrt{\frac{1}{a_{0}-1}}, \lambda_{0}=-1-\frac{1}{\tilde{\tau}}$. Substituting these values in (12) allows to the value of $\alpha$. Simple computations shows that multiplicity four is not possible since the incompatibility of the obtained solution with the vanishing of the third derivative, which concludes ii). The proof of iii) is similar to the one proposed in [7] and [13]. By substituting the parameters' values guaranteeing such a maximal multiplicity, one writes the corresponding equation as:

$$
\tilde{\Delta}(\lambda, \tilde{\tau})=\left(\lambda-\lambda_{0}\right)^{2}\left(1+\frac{\tilde{\Delta}(\lambda, \tilde{\tau})-\left(\lambda-\lambda_{0}\right)^{2}}{\left(\lambda-\lambda_{0}\right)^{2}}\right)=0 .
$$

Writing explicitly the expression of $\tilde{\Delta}$ in (14) then substituting the parameter values from (11) gives:

$$
\tilde{\Delta}(\lambda, \tilde{\tau})=\left(\lambda-\lambda_{0}\right)^{2}\left(1-2 \int_{0}^{1} \int_{0}^{s} \mathrm{e}^{-\tilde{\tau}\left(\lambda-\lambda_{0}\right) t} d t d s\right)=0 .
$$

Let assume that $\lambda_{1}=\gamma_{1}+j \omega_{1}$ is a root of (10) such that $\gamma_{1}>z_{0}$, then $\lambda_{1}$ should be a root of the second factor of $\tilde{\Delta}$ in. Thus,

$$
\begin{aligned}
1 & =2 \int_{0}^{1} \int_{0}^{s} \mathrm{e}^{-\tilde{\tau}\left(\lambda-\lambda_{0}\right) t} d t d s=2\left|\int_{0}^{1} \int_{0}^{s} \mathrm{e}^{-\tilde{\tau}\left(\lambda-\lambda_{0}\right) t} d t d s\right| \\
& \leq 2 \int_{0}^{1} \int_{0}^{s}\left|\mathrm{e}^{-\tilde{\tau}\left(\lambda-\lambda_{0}\right) t}\right| d t d s
\end{aligned}
$$

But $\gamma_{1}>z_{0}$ then $\left|\mathrm{e}^{-\tilde{\tau}\left(\lambda-\lambda_{0}\right) t}\right|<1$, which shows the inconsistency of such an assumption. This concludes that $z_{0}$ is the rightmost root of $\tilde{\Delta}$. 


\section{Remark 1.}

- In terms of the initial equation (8) parameters', the only admissible triple root in the Laplace variable $s$ is given by $s_{0}=\frac{c_{1}}{2} \lambda_{0}$. Furthermore, if such a configuration holds then $s_{0}$ is the rightmost root corresponding to (8). If in addition the delay $\tau$ satisfy $c_{1} \tau+2>0$ then the trivial solution corresponding to (8) is asymptotically stable.

- In the case of a real non semi-simple spectral value (with an algebraic multiplicity $n$ greater than 1 and a geometric multiplicity 1) corresponding to the complete regular splitting, using a perturbation approach, it is shown in [19] that the behavior of a real eigenvalue with multiplicity $n$, corresponds to a minimum of the spectral abscissa function, as a function of a delay parameter.

- The use of the delayed feedback is in general motivated by the simplicity of the design procedure as well as the controller implementation. Furthermore, in [17] the delay stabilizing effect is emphasized where the explicit conditions on the pair $(\alpha, \tau)$ are established such that the delayed feedback stabilizes the plant, but the closed loop system would be instable if the delay $\tau$ is set to zero.

\section{Application to Vibration control}

The problem of active vibration damping of thin mechanical structures is a topic that has received great attention by the control community for several years [20], especially, when actuators and sensors are based on piezoelectric materials. For mechanical structures that are deformable, piezoelectric materials are used as strain sensors or strain actuators. With an appropriate controller, they allow to achieve shape control [e.g. 21, 22]) or the active damping of multi-modal vibrations thanks to their very large bandwidth. Moreover, their behavior is quite linear when they work in a specific range of use. This explains in part the great interest of using piezoelectric materials for the instrumentation of thin mechanical structures. In this area, the major challenge is the design of controllers able to damp the most vibrating modes in a specified low-frequency bandwidth while ensuring robustness against high-frequency modes, outside the bandwidth of interest, often unmodelled or weakly modelled. The inherent feature of this kind of systems is that they arise robustness issues when they are tackled with finite dimensional control tools. Many works have concerned the vibration control problem of the "Euler-Bernoulli beam" equipped with one rectangular piezoelectric actuator and sometimes, another one, identical and collocated, but used as sensor, see for example [23], [24] where one edge of the beam is clamped whereas the other remains free. Other works dealt with the problem of vibration control for laminated rectangular plates [25] or complex plate like structures [26].

In this work, we consider the flexible structure depicted in Fig. 1. It is an aluminium-based beam, embedded in a mobile support. The mobile support is subjected to an acceleration, denoted by $w$ in the sequel, and it is moving along the $z$ axis. This flexible beam is equipped with two lead zirconate titanate piezoelectric patches (also called PZT): one of them is used as an actuator and the other works as a sensor. These patches are supposed to be rigidly bounded on the beam, one on each side, located at the clamped edge. The whole device is designated hereafter as a piezo-actuated beam. It can be deformed by the application of a voltage, denoted by $u$, across the actuator. The sensor delivers an electrical voltage which corresponds to a measure, denoted by $y$, of the local deformation under the piezoelectric patch. 


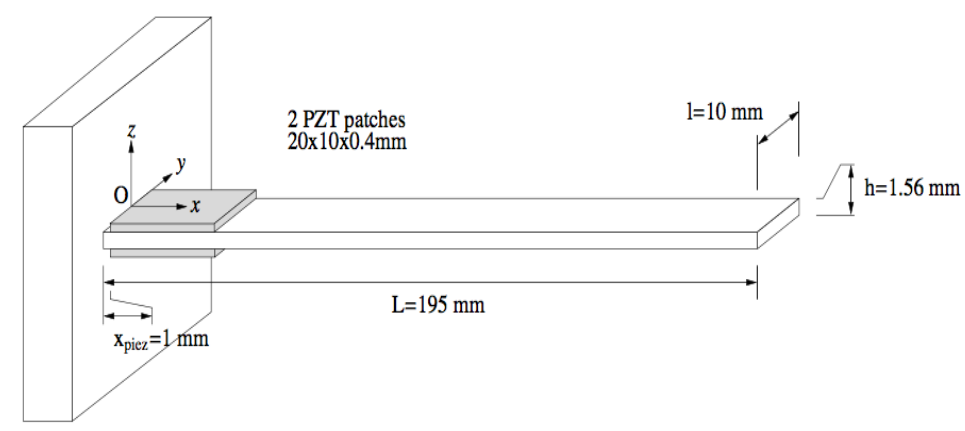

Figure 1: Sketch of the piezo-actuated flexible beam, clamped at one edge.

Very often, this equipped mechanical structure is partly described by the in-plane EulerBernoulli Partial Differential Equation (PDE) that suffers from the lack of precision in describing the electro-mechanical interactions between the passive structure and the piezoelectric components. Indeed, these latter are often withdrawn in the computation of the eigenfrequencies [27] of the whole structure. Nevertheless, such a structure obeys to fundamental equations of continuum mechanics in 3D space [28], involving computations of gradient of displacement vector and divergence operator applied to strength tensor. When completed with Neumann and Dirichlet boundary conditions, the fundamental equations give several PDEs that are coupled, thus that are impossible to solve analytically. Then, engineers that need inputs-to-outputs dynamical models naturally watch for numerical methods [29].

Finite Element Modelling (FEM) is a numerical method that approximates the displacement field with a displacement vector of finite dimension, denoted $q \in \mathbb{R}^{n_{\text {dof }}}$, containing as many components as needed to describe the displacement field in the 3D space, for both mechanical and electrical variables [30]. This method leads to the following matricial Ordinary Differential Equations (ODE), time invariant but coupled and linear [29]:

$$
\begin{aligned}
\mathbb{M}_{q q} \ddot{q}(t) & +\mathbb{D}_{q q} \dot{q}(t)+\mathbb{K}_{q q} q(t)=\mathbb{M}_{q w} w(t)-\mathbb{K}_{q u} u(t) \\
y(t) & =\mathbb{K}_{q y} q(t) \\
z(t) & =\mathbb{F}_{z w} w(t)-\mathbb{F}_{z u} u(t)-\mathbb{F}_{z q} q(t)-\mathbb{F}_{z v} \dot{q}(t)
\end{aligned}
$$

where $w(t) \in \mathbb{R}$ is the absolute acceleration $\left(\mathrm{m} / \mathrm{s}^{2}\right)$ of the movable support along axis $z, z(t) \in \mathbb{R}$ is the relative acceleration $\left(\mathrm{m} / \mathrm{s}^{2}\right)$ of the free end, derived from the equations of motion, $u(t) \in \mathbb{R}$ is the piezoelectric voltage $(V)$ across the actuator (control signal), $y(t) \in \mathbb{R}$ is the piezoelectric sensor voltage $(V)$ across the sensor (measured output signal $y(t)$ ). Moreover, the terms $\mathbb{M}_{q q}, \mathbb{D}_{q q}$, $M_{q w}, \mathbb{K}_{q q}, \mathbb{K}_{q u}, \mathbb{K}_{q y}, \mathbb{F}_{z w}, \mathbb{F}_{z u}, \mathbb{F}_{z q}$ and $\mathbb{F}_{z v}$ are all matrices derived from the assembly step of the FEM such that $q(t) \in \mathbb{R}^{1000}$, i.e. several thousands degrees of freedom. The dynamical model given by equations (17) - (19) is not numerically tractable because of the very large number of degrees of freedom $n_{\text {dof }} \approx 10^{3}$ for our flexible system.

After producing a FEM, a modal analysis is performed to the undamped motion equation (17). It consists in finding the eigenstructure of

$$
\mathbb{M}_{q q} \ddot{q}(t)+\mathbb{K}_{q q} q(t)=0,
$$

in order to use the eigenvectors as a new basis in which equations (17)—(19) form a new system 
of ODEs, still linear but decoupled, involving a new state vector, called vector of modes. The advantage of this framework is that it allows to build a model in state-space form devoted to the analysis of (20) with an order sufficiently small to describe the dynamical behavior within a lowfrequency bandwidth. Furthermore, a reduced order system, devoted to the synthesis step, is also available and differs from (20) by the presence of feedthrough terms between output $y$ and inputs $w$ and $u$. On the one hand, the analysis model is of order 12, containing two uncontrollable and unobservable modes, and describing the inputs-outputs behavior in the bandwidth $[0-3500 \mathrm{~Hz}]$. On the other hand, the reduced system is of order 2 , including the first bending mode. This mode is controllable and observable. Let $x_{p} \in \mathbb{R}^{n_{p}}$ be the state vector of the system, whatever its order, meaning either $n_{p}=12$ for the analysis model or $n_{p}=2$ for the synthesis model. The frequency responses are shown in Fig. 2 and the shapes of the first bending modes in Fig. 3.

$$
\mathcal{P}\left\{\begin{aligned}
\dot{x}_{p}(t) & =A_{p} x_{p}(t)+B_{p, w} w(t)+B_{p, u} u(t) \\
z(t) & =C_{p, z} x_{p}(t)+D_{p, z w} w(t)+D_{p, z u} u(t) \\
y(t) & =C_{p, y} x_{p}(t)+D_{p, y w} w(t)+D_{p, y u} u(t)
\end{aligned}\right.
$$

It is worth mentioning that the piezo-actuated beam is a SISO system, i.e. with only one actuator,
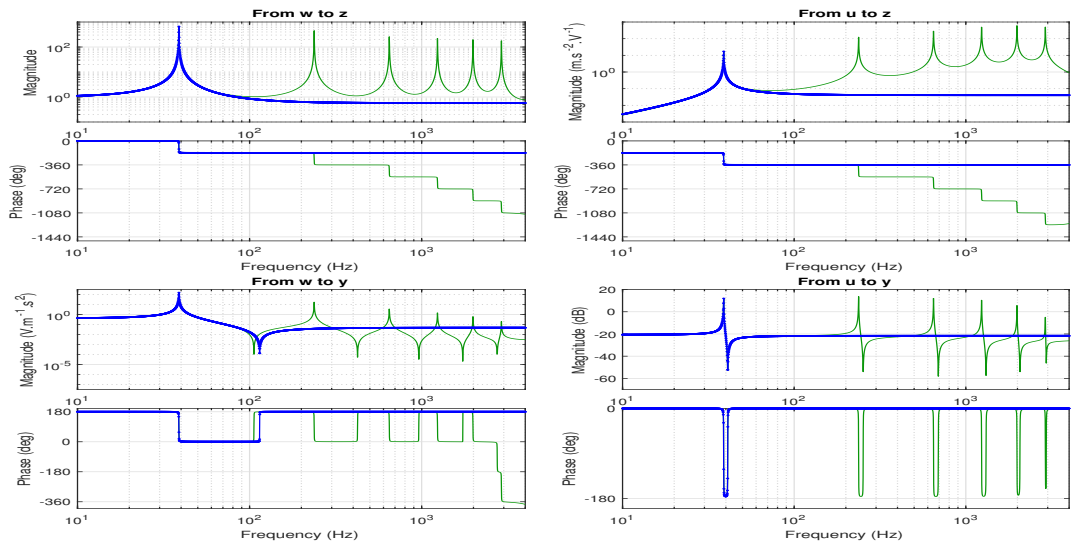

Figure 2: Analysis (-) and synthesis (.-) models' frequency responses

driven by the controlled electrical voltage $u$, and only one measured output signal corresponding to the electrical voltage $y$. The perturbation input $w$ is the total acceleration applied to the clamped edge of the structure. The vertical total acceleration of the free edge is our controlled output $z$. An interesting control objective would be to damp the peak of resonance of the first bending mode, by using an output feedback controller of low gain, in order to avoid the control signal saturation in closed-loop.

\section{Vibration damping}

The transfer function corresponding to the first mode of vibration is given by:

$$
G(s)=\frac{N(s)}{D(s)},
$$



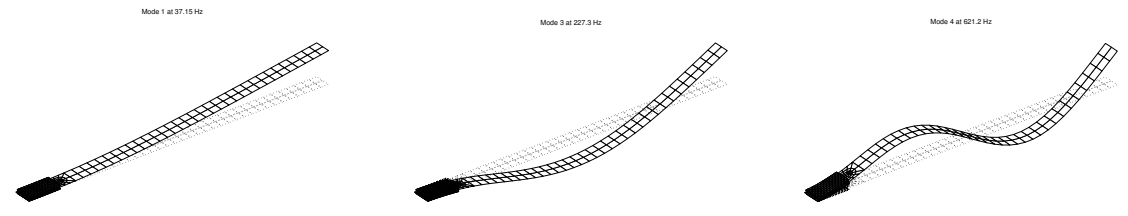

Figure 3: Three first controllable and observable modes.

with the following numerical expressions of $N$ and $D$ which are derived from the reduced-order state-space model briefly described in Section 2:

$$
\left\{\begin{array}{l}
N(s)=0.0824705565013658 s^{2}+0.0402320642368774 s+5472.41008648971 \\
D(s)=s^{2}+0.487835488732404 s+59495.8660165543
\end{array}\right.
$$

Since we are considering the configuration where the sensor and the actuator are collocated, then the roots of the second order polynomial $N(s)$ are with negative real parts as indicated in [31].

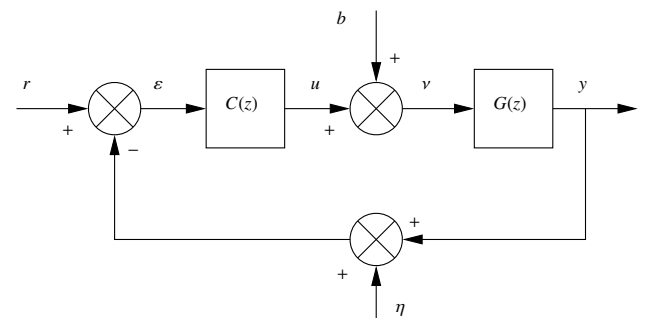

Figure 4: Feedback control structure for active vibration damping.
Consider now the piezo-actuated system inserted in the typical output feedback control structure of Fig. 4, with a reference signal $r$ equal to zero, no additive input disturbance $b$ and no additive output disturbance $\eta$. We define the following controller given in the Laplace domain by

$$
C(s)=\frac{\beta e^{-\tau s}}{N(s)} .
$$

Using the normalization (9), the closed-loop characteristic function takes the form (10). Following Theorem 3.1, one has, a dominant root of multiplicity 3 at $\lambda_{0}=-1000.999500$ for an admissible delay value $\tilde{\tau}=0.001000000500$ and the gain value $\alpha=-735022.75582638422906$.

\section{Comparison with a linear pole placement controller}

In order to show the effectiveness of the approach, we propose to design a linear controller derived from the pole placement approach and compare the time responses of both controllers, in closed-loop, to a disturbance $w$ corresponding to a square tooth signal. To have a relevant comparison, the Pole-Placement (PP) linear controller is designed to assign all the closed-loop poles in the same location of the complex plane, and with the same algebraic multiplicity, as the multiple pole obtained with the Filtered Delayed Proportional (FDP) controller. Let us set $N(s)=b_{2} s^{2}+b_{1} s+b_{0}$ and $D(s)=s^{2}+c_{1} s+c_{0}$ and define the desired closed-loop characteristic function with the pole-placement controller as

$$
\Pi_{d}(s)=\left(s-s_{0}\right)^{3}
$$


where $s_{0}=\lambda_{0} c_{1} / 2$ is the multiple pole obtained with the filtered delayed proportional controller. The pole-placement controller is sought as the following rational fraction

$$
C_{p p}(s)=\frac{S(s)}{R(s)}
$$

where the degrees of the polynomials $S(s)$ and $R(s)$ respectively $\sigma$ and $\rho$ satisfy $\sigma \leq \rho$. Now define $\tilde{R}(s):=R(s)+b_{2} S(s)$ as a monic polynomial of degree $\tilde{\rho}$. So that the pole-placement controller which is obtained by solving the following Diophante equation, in the unknown polynomials $S$ and $R$,

$$
D(s) R(s)+N(s) S(s)=\Pi_{d}(s)
$$

is equivalent to this solving other Diophante equation, in the unknown polynomials $S$ and $\tilde{R}$

$$
D(s) \tilde{R}(s)+\tilde{N}(s) S(s)=\Pi_{d}(s)
$$

where $\tilde{N}(s):=N(s)-b_{2} D(s):=\tilde{b}_{1} s+\tilde{b}_{0}$ is of degree 1. Since the degrees of $N(s)$ and $D(s)$ are both equal to 2, it can be shown that the minimal value of $\rho$ and $\sigma$ that fulfills the constraint is $\rho=\sigma=1$. In that case, we set $\tilde{R}(s)=s+\tilde{r}_{0}$ and $S(s)=s_{1} s+s_{0}$ and (26) is equivalent to the following linear system

$$
\left[\begin{array}{ccc}
1 & \tilde{b}_{1} & 0 \\
c_{1} & \tilde{b}_{0} & \tilde{b}_{1} \\
c_{0} & 0 & \tilde{b}_{0}
\end{array}\right]\left[\begin{array}{c}
\tilde{r}_{0} \\
s_{1} \\
s_{0}
\end{array}\right]=\left[\begin{array}{c}
-3 s_{0}-c_{1} \\
3 s_{0}^{2}-c_{0} \\
-s_{0}{ }^{3}
\end{array}\right] .
$$

Numerically, we obtain $R(s) \simeq-16.34556626 s+4958.655183$ and $S(z) \simeq 210.3243509 s-$ 51250.51385 . Figure 5 shows the time responses of the measured output $y$ when the beam system is submitted to a disturbance $w$ similar to a shock applied at the clamped end. Three responses are shown: the gray one is the response when no controller is applied (open-loop response); the red one is the response when the PP controller is applied and the blue one is the response when the FDP controller is applied. Firstly, both controllers PP and FDP manage to suppress the vibrations quickly with roughly the same dynamic. Secondly, one notes that the peak at the beginning of the response is higher in the case of the PP controller, meaning that the FDP controller enhance this behavior with respect to the PP controller. Figure 6 shows the time responses of the closed-loop voltage across the piezoelectric actuator (control signal), when the beam system is still submitted to the same disturbance $w$. One notes that the PP controller develops a peak of voltage higher than the FDP controller, whereas this last one oscillates longer in time.

\section{Conclusion}

In this paper, we illustrated the interest of using time-delay in the controller design as a control parameter. The applicability of the rightmost root assignment in the problem of vibration's attenuation in the case of a single vibrating mode was also emphasized. In future works, the proposed controller design will be applied to further vibrating modes and it will therein be compared with other optimal controllers of finite dimension. 


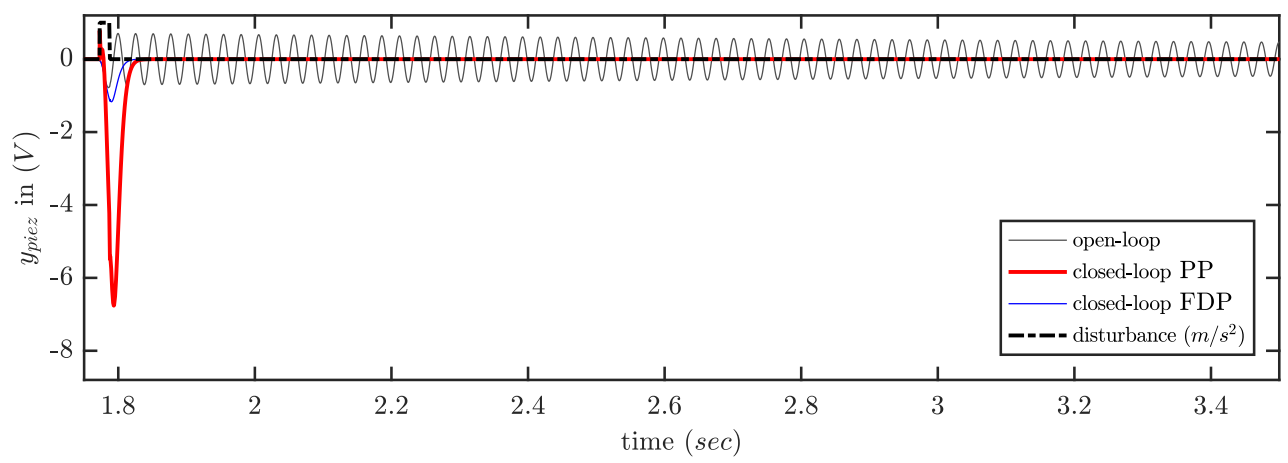

Figure 5: Time responses of the piezoelectric sensor in open and closed-loop.

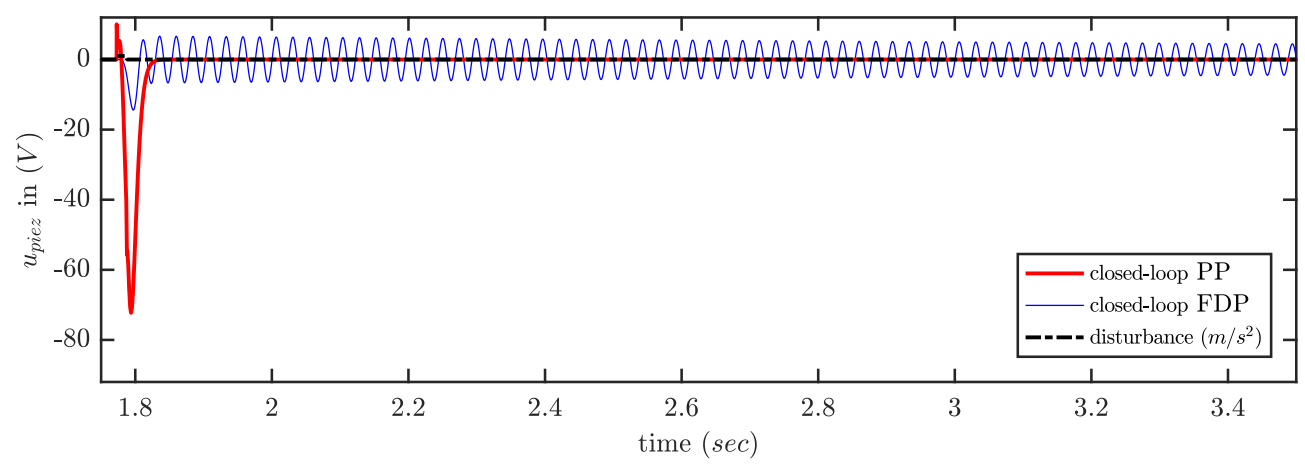

Figure 6: Time responses of the voltage across the piezoelectric actuator in closed-loop.

Acknowledgements. The authors would like to thank the reviewers for valuable suggestions. We warmly thank Professor Gábor Stépán (Budapest University of Technology and Economics, Hungary) for stimulating discussions on delay topics. Last but not least, we thank Karim L. Trabelsi (IPSA Paris, France) for careful reading of the manuscript and for valuable remarks. IB and SIN are partially financially supported by a grant from Hubert Curien (PHC) BRANCUSI 2017, project number 38390ZL.

\section{References}

[1] R. Bellman, K. Cooke, Differential-difference equations, Academic Press, New York, 1963.

[2] B. Levin, Distribution of zeros of entire functions, Translations of Mathematical Monographs, AMS, Providence, Rhode Island, 1964.

[3] W. Michiels, S.-I. Niculescu, Stability and stabilization of time-delay systems, Vol. 12 of Advances in Design and Control, Society for Industrial and Applied Mathematics (SIAM), 2007.

[4] W. Govaerts, J. Guckenheimer, A. Khibnik, Defining functions for multiple hopf bifurcations, SIAM Journal on Numerical Analysis 34 (3) (1997) 1269-1288.

[5] M. Dellnitz, B. Werner, Computational methods for bifurcation problems with symmetries-with special attention to steady state and hopf bifurcation points, Journal of Computational and Applied Mathematics 26 (1-2) (1989) 97 -123 . 
[6] J. Vanbiervliet, K. Verheyden, W. Michiels, S. Vandewalle, A nonsmooth optimisation approach for the stabilisation of time-delay systems, ESAIM: COCV 14 (3) (2008) 478-493.

[7] I. Boussaada, S. I. Niculescu, Tracking the algebraic multiplicity of crossing imaginary roots for generic quasipolynomials: A Vandermonde-based approach, IEEE Transactions on Automatic Control 61 (2016) 1601-1606.

[8] I. Boussaada, S.-I. Niculescu, Characterizing the codimension of zero singularities for time-delay systems, Acta Applicandae Mathematicae 145 (1) (2016) 47-88.

[9] I. Boussaada, S.-I. Niculescu, Computing the codimension of the singularity at the origin for delay systems: The missing link with Birkhoff incidence matrices, 21st International Symposium on Mathematical Theory of Networks and Systems (2014) $1-8$.

[10] G. Pólya, G. Szegő, Problems and Theorems in Analysis, Vol. I: Series, Integral Calculus, Theory of Functions, Springer-Verlag, New York, Heidelberg, and Berlin, 1972.

[11] A. Ramirez, S. Mondie, R. Garrido, R. Sipahi, Design of proportional-integral-retarded (pir) controllers for secondorder lti systems, IEEE Transactions on Automatic Control (99) (2015) 1-6.

[12] T. Vyhlidal, P. Zitek, Mapping based algorithm for large-scale computation of quasi-polynomial zeros, IEEE Transactions on Automatic Control 54 (1) (2009) 171-177.

[13] I. Boussaada, H. Unal, S.-I. Niculescu, Multiplicity and stable varieties of time-delay systems: A missing link, in: Proceeding of the 22nd International Symposium on Mathematical Theory of Networks and Systems, 2016, pp. $1-6$.

[14] I. Boussaada, S.-I. Niculescu, S. Tliba, T. Vyhlidal, On the coalescence of spectral values and its effect on the stability of time-delay systems: Application to active vibration control, Procedia IUTAM 22 (Supplement C) (2017) 75 - 82, IUTAM Symposium on Nonlinear and Delayed Dynamics of Mechatronic Systems.

[15] K. L. Cooke, Stability analysis for a vector disease model, Rocky Mountain J. Math. 9 (1979) 31-42.

[16] S. Ruan, Delay differential equations in single species dynamics, in: Delay Differential Equations and Applications, Vol. 29 of Fields Inst. Commun., Springer, Berlin, 2006, pp. 477-517.

[17] S.-I. Niculescu, W. Michiels, K. Gu, C. T. Abdallah, Delay Effects on Output Feedback Control of Dynamical Systems, Springer Berlin Heidelberg, Berlin, Heidelberg, 2010, pp. 63-84.

[18] O. Kirillov, M. Overton, Robust stability at the swallowtail singularity, Frontiers in Physics 1 (2013) 24.

[19] W. Michiels, I. Boussaada, S. Niculescu, An explicit formula for the splitting of multiple eigenvalues for nonlinear eigenvalue problems and connections with the linearization for the delay eigenvalue problem, SIAM J. Matrix Analysis Applications 38 (2) (2017) 599-620.

[20] S. Tliba, Control of a Vibrating Axisymmetric Membrane Using Piezoelectric Transducers, in: 18th IFAC World Congress, Milano, Italy, 2011, p. 7713 (6 pages).

[21] E. Park, J. Mills, Static shape and vibration control of flexible payloads with applications to robotic assembly, IEEE/ASME Trans. on Mechatronics, 10 (6) (2005) 675-687.

[22] W. Juan, H. Hongsheng, Q. Suxiang, Q. Linfang, Research on shape control and active vibration control of piezoelectric composite laminated shell, in: ICEMI '07. 8th International Conference on Electronic Measurement and Instruments, 2007., 2007, pp. 4-569-4-576.

[23] W. Chen, M. Buehler, G. Parker, B. Bettig, Optimal sensor design and control of piezoelectric laminate beams, IEEE Trans. on Control Systems Technology, 12 (1) (2004) 148-155.

[24] H. Banks, R. del Rosario, H. Tran, Proper orthogonal decomposition-based control of transverse beam vibrations experimental implementation, IEEE Trans. on Control Systems Technology, 10 (5) (2002) 717-726.

[25] M. Kögl, M. L. Bucalem, Analysis of smart laminates using piezoelectric mitc plate and shell elements, Computers \& Structures 83 (2005) 1153-1163.

[26] S. Tliba, H. Abou-Kandil, C. Prieur, Active vibration damping of a smart flexible structure using piezoelectric transducers: $\mathcal{H}_{\infty}$ design and experimental results, in: Proc. of the 16th IFAC World Congress, IFAC, Prague, 2005.

[27] S. Tliba, Contrôle actif des vibrations dans des structures mécaniques minces instrumentées de transducteurs piézoélectriques, Thèse de doctorat (in french), École Normale Supérieure de Cachan, Cachan (94), France (Décembre 2004).

[28] M. Gérardin, D. Rixen, Mechanical vibrations: theory and application to structural dynamics, 2nd Edition, WILEY, England, 1997.

[29] L. Komzsik, What every engineer should know about computational techniques of finite element analysis, CRC Press, Taylor \& Francis Group, NW, FL, 2005.

[30] A. Benjeddou, Advances in piezoelectric finite element modeling of adaptative structural elements: a survey, Computers \& Structures 76 (2000) 347-363.

[31] A. Preumont, Vibration Control of Active Structure, An Introduction, 2nd Edition, Solid Mechanics and its Applications, Kluwer Academic Publishers, Dordrecht / Boston / London, 2002. 


\section{Appendix A: Pólya-Szegö Theorem}

Theorem 7.1 (Pólya-Szegö, [10], pp. 144). Let $\tau_{1}, \ldots, \tau_{N}$ denote real numbers such that

$$
\tau_{1}<\tau_{2}<\ldots<\tau_{N}
$$

and $d_{1}, \ldots, d_{N}$ positive integers satisfying

$$
d_{1} \geq 1, d_{2} \geq 1 \ldots d_{N} \geq 1, \quad d_{1}+d_{2}+\ldots+d_{N}=D+N .
$$

Let $\sharp$ be the number of zeros of the function

$$
f(s)=\sum_{1 \leq i \leq N, 1 \leq j \leq d_{i}} c_{i, j} f_{i, j}(s)
$$

that are contained in the horizontal strip $\alpha \leq \mathcal{I}(z) \leq \beta$ where $f_{i, j}(s)=s^{j-1} e^{\tau_{i} s}$, for $1 \leq j \leq d_{i}$ and $1 \leq i \leq N$.

Assuming that

$$
\sum_{1 \leq k \leq d_{1}}\left|c_{1, k}\right|>0, \ldots, \sum_{1 \leq k \leq d_{N}}\left|c_{N, k}\right|>0
$$

then

$$
\frac{\left(\tau_{N}-\tau_{1}\right)(\beta-\alpha)}{2 \pi}-D+1 \leq \sharp \leq \frac{\left(\tau_{N}-\tau_{1}\right)(\beta-\alpha)}{2 \pi}+D+N-1 .
$$

Setting $\alpha=\beta=0$, the above theorem yields $\sharp_{P S} \leq D+N-1$ where $D$ stands for the sum of the degrees of the polynomials involved in the quasipolynomial function $f$ and $N$ designates the associated number of polynomials. This gives a sharp bound for the number of $f$ 's real roots. 